\title{
Interrelations between the pairing and quadrupole interactions in the microscopic Shell Model
}

\author{
K. P. Drumev ${ }^{1, a}$ and A. I. Georgieva ${ }^{2, b}$ \\ ${ }^{1}$ Institute for Nuclear Research and Nuclear Energy, Bulgarian Academy of Sciences, Sofia 1784, Bulgaria \\ ${ }^{2}$ Institute of Solid State Physics, Bulgarian Academy of Sciences, Sofia 1784, Bulgaria
}

\begin{abstract}
The symmetry-adapted Pairing-plus-Quadrupole Model (PQM) is explored in the framework of the Elliott's SU(3) Model with the aim to obtain the complementary and competing features of the pairing and quadrupole interactions in the model Hamiltonian, containing both of them as limiting cases or dynamical symmetries. The probability distribution of the $\mathrm{SU}(3)$ basis states within the $\mathrm{SO}(8)$ pairing states is obtained through a numerical diagonalization of the PQM Hamiltonian. In an application of the model for the description of the ${ }^{20} \mathrm{Ne}$ and ${ }^{20} \mathrm{O}$ spectra, we investigate systematically the relative strengths of dynamically symmetric quadrupole-quadrupole interaction with the isoscalar, isovector and total pairing interactions. The approach allows for an extension of the model space for two oscillator shells and introduction of more elaborate pairing interaction. The new effects that come with this generalization will also be discussed as a future development.
\end{abstract}

\section{Introduction}

The algebraic realization of the dynamical symmetries that appear in the microscopic shell model was presented in Refs. [1, 2]. In it the dynamical symmetries of three types of pairing interactions in addition to the quadrupole-quadrupole interaction, introduced through Elliott's SU(3) model were defined as different phases of the microscopic shell model and the phase transitions between theme were investigated by means of introducing control parameters in a generalized Hamiltonian, containing the interactions of the considered two or three limiting cases. Here, we aim at exploiting the applications of the theory in some realistic nuclear systems. In order to evaluate the limits of application of this approach, we start with the simplest real test case - the $d s$-shell, which is the first one, where both deformation and pairing phenomena play an important role [3, 4]. Our proof-of-case example presents the simple but complete systems of 2 and 4 particles in the $d s$-shell which allows us to study the Pairingplus-Quadrupole Model (PQM) without any truncation of the model space.

We first present results for the total-pairing eigenstates with the idea to underline the importance of only a few $\mathrm{SU}(3)$ irreducible representations. We also show the outcome for the excitation spectra, as well as a study of the interrelation between the pairing and the quadrupole interactions. The excitation spectrum characteristics are demonstrated to change from the very degenerated pairinglike pattern to the rotational-like spectrum of the pure quadrupole-quadrupole interaction. We also evaluate the

\footnotetext{
a e-mail: kdrumev2000@yahoo.com

be-mail: anageorg@issp.bas.bg
}

contribution from the addition of one more free parameter, namely separating the isovector from the isoscalar pairing mode. The results show an improvement of the description of the low-lying collective spectra of the considered realistic nuclear systems. By representing the best-fit results for these systems on the symmetry triangle of the phase diagram, we illustrate which of the interactions are more likely to prevail in the real nuclei.

\section{Description of the calculations}

In this contribution, we present the application of the dynamical symmetries that were established in the Microscopic Shell Model in the $d s$-shell for the even-even nuclei with 2 valence particles: ${ }^{18} \mathrm{Ne}$ and ${ }^{18} \mathrm{O}$, and with 4 valence particles: ${ }^{20} \mathrm{Ne}$ as well as ${ }^{20} \mathrm{O}$. The following observables are evaluated for the spectra of these nuclei: the root mean square (RMS) deviation of the model energies from the experimental ones, the weight of each of the isoscalar, isovector and quadrupole interactions in the correct reproduction of the experiment. Also, information for the structure of the wave-function and/or $B(E 2)$ transitions may be added for best-fit values. We also aim to improve our bestfit results for the two-parameter Hamiltonian by considering the three-parameter case. The nucleus ${ }^{20} \mathrm{Mg}$ is excluded from our calculation because of not enough experimental states to be described.

To do our calculations, we work in the SU(3) basis

$$
\left|\Psi_{R}\right\rangle \equiv\left|\{f\} \alpha(\lambda, \mu) \kappa L, S ; J M_{J}\right\rangle
$$

labeled by the representations of the dynamical symmetry:

$$
\begin{array}{lllc}
\mathrm{U}(\Omega) \supset & \mathrm{SU}(3) \supset & \mathrm{SO}(3) \\
\{f\} & \alpha & (\lambda, \mu) & \kappa L
\end{array}
$$


We generate it by using the rules of $U(\Omega)$ to $S U(3)$ reduction (tabulated in the code [5]). We also rely on tools developed to calculate reduced matrix elements for any type of physical operator between different SU(3) irreps [6]. We calculate the matrix elements of all the operators in this basis and then perform a numerical diagonalization to obtain the energy spectrum and the eigenstates.

The Hamiltonian we use for studying the phase transitions consists of pairing and quadrupole terms. In the case of two parameters, it has the form

$$
V_{\text {res }}=\frac{1}{2}(1-x) V_{1}+\frac{1}{2}(1+x) V_{2},
$$

where at $x=-1$ we have pure $V_{1}$ interaction and at $x=1$ the limiting case of pure $V_{2}$ interaction is realized. Compared to the earlier SU(3) one-shell realization [4, 7] of the PQM, we use a more general pairing Hamiltonian which includes proton-neutron pairing terms as well.

\section{Relation between the pairing and the $\mathrm{SU}(3)$ basis states}

The decomposition of the total pairing basis states over the $\mathrm{SU}(3)$ basis states $\left|\Psi_{P}\right\rangle_{i} \equiv$ $\left|\{f\}, v\left[p_{1}, p_{2}, p_{3}\right] \beta L, S ; J M_{J} T M_{T}\right\rangle_{i} \equiv\left|\{f\}, i, L, S ; J M_{J}\right\rangle=$ $\sum_{j} C_{i j}\left|\{f\}, j, L, S ; J M_{J}\right\rangle=\sum_{j} C_{i j}\left|\Psi_{R}\right\rangle_{j}$ can be done by calculating the matrix elements of the pairing terms which enter the equation

$$
\begin{array}{r}
\left\langle\Psi_{P}\left|H_{\text {pair }}\right| \Psi_{P}\right\rangle=E_{\text {pair }}(m, i,[P],(S T))= \\
\sum_{j k} C_{k i}^{*} C_{i j} . \delta_{k j \cdot k}\left\langle\Psi_{R}\left|H_{\text {pair }}\right| \Psi_{R}\right\rangle_{j} .
\end{array}
$$

from [2]. Example of this decomposition of the first few low-lying pairing states into $\mathrm{SU}(3)$ basis states is given in Table 1. From this decomposition one can extract information on the intrinsic deformation of the pairing states through the content in percentage of the SU(3) states, which are clearly associated with the $(\beta \gamma)$ variables of the Geometric Collective Model [8]. As could be seen from Table 1 and Fig. 1, for the yrast states the prolate components $(8,0)$ and $(4,2)$ play a dominant role, although for the ground state they are strongly mixed with the oblate $(0,4)$ state.

The energy of the pairing states is given by Eq. (20) in Ref. [9] where a complete classification of the states in the $\mathrm{SO}(8)$ proton-neutron pairing model has been done.

\section{Two-parameter estimate}

We start with the two parameter interaction:

$$
H=\frac{(1-x)}{2}\left[G_{0} P_{\mathrm{isc}}+G_{1} P_{\mathrm{iv}}\right]-\frac{\chi}{2}(1+x) Q \cdot Q
$$

with $G_{0}=G_{1}$,

$$
\begin{aligned}
& H=\frac{(1-x)}{2} G_{0} P_{\mathrm{isc}}-\frac{\chi}{2}(1+x) Q \cdot Q \\
& H=\frac{(1-x)}{2} G_{1} P_{\mathrm{iv}}-\frac{\chi}{2}(1+x) Q \cdot Q
\end{aligned}
$$
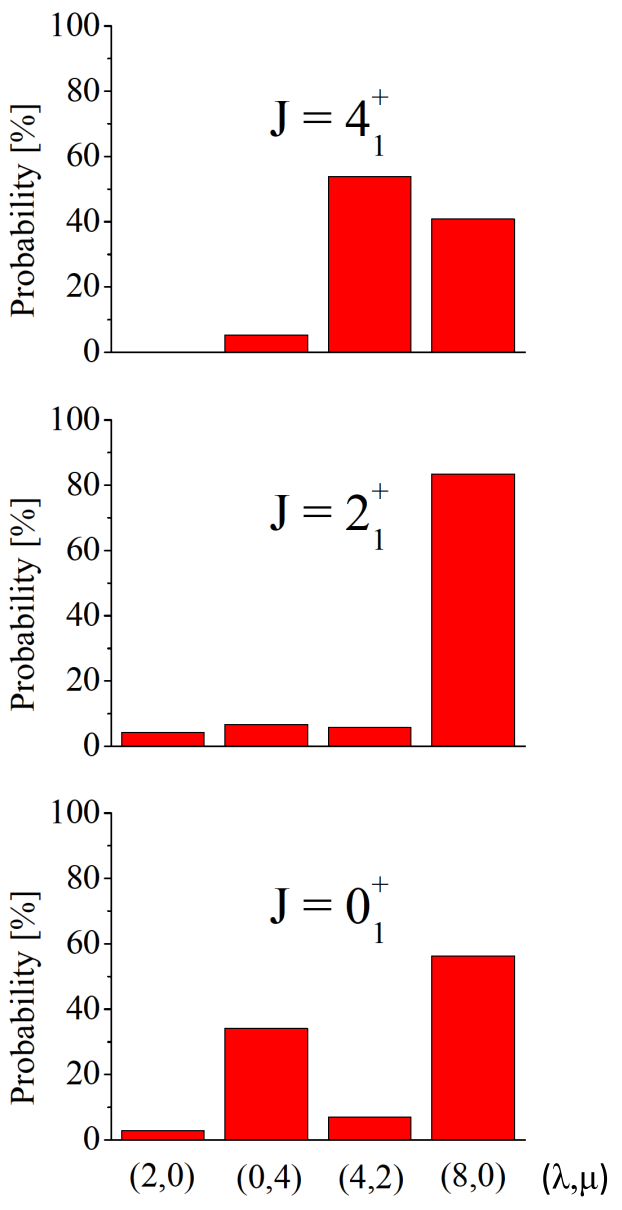

Figure 1. (Color online) The structure of the pairing states for the system of 2 protons and 2 neutrons in the $d s$-shell.

for the cases when both pairing modes are present with the same strength or when we have just the isoscalar or the isovector mode involved, respectively.

Now, let us use the energies of the low-lying states of ${ }^{20} \mathrm{O}$, which has 4 neutrons in the $d s$ valence shell. In Fig. 2, we present the results of a minimization procedure for the root-mean-squared value $\sigma=\sqrt{\sum_{i}\left(E_{T h}^{i}-E_{E x p}^{i}\right)^{2} / d}$ ( per degree of freedom $d$ ) with respect to the two parameters $G$ and $\chi$ of the residual interaction (5). The darker spots in the middle of the figure present the intervals of change of the parameters for which we have the minimal values of $\sigma$ or the values of the parameters fitted to a set of the 12 lowest-lying positive-parity experimental energies $E_{E x p}^{i}$ from the observed spectra [10] of a real nuclear system. The red dashed line in Fig. 2(a) connects the values of each of the parameters $G$ and $\chi$ at their respective limiting cases of pure pairing or pure quadrupole-quadrupole interactions. This line could be assigned as the axis of change of the parameter $-1 \leqslant x \leqslant 1$ as is used in Fig. 2(b). The regions of the optimal values for the parameters lie on this line and their position in respect to its center could 
Table 1. Decomposition of the total pairing states $\left|\Psi_{P}\right\rangle$ for the nuclear system of 2 protons and 2 neutrons in the $d s$-shell in terms of $\mathrm{SU}(3)$ basis states $\left|\Psi_{R}\right\rangle$. In the first column, the $J^{\pi}$ value of the pairing state is given.

\begin{tabular}{|c|c|c|c|}
\hline$|i\rangle \equiv\{|v[p][P] L, S ; J\rangle\}$ & Energy[MeV] & $\mid\{j\} \equiv\{(\lambda, \mu) L, S ; J\rangle\}\rangle$ & $\left|C_{i j}\right|^{2}[\%]$ \\
\hline \multirow{4}{*}{$\begin{array}{c}0_{1}^{+} \\
|0[0][0] 0,0 ; 0\rangle\end{array}$} & -16 & $(8,0) 0,0 ; 0$ & 56.25 \\
\hline & & $(4,2) 0,0 ; 0$ & 6.94 \\
\hline & & $(0,4) 0,0 ; 0$ & 34.03 \\
\hline & & $(2,0) 0,0 ; 0$ & 2.78 \\
\hline \multirow{4}{*}{$\begin{array}{c}2_{1}^{+} \\
|2[1][0] 2,0 ; 2\rangle\end{array}$} & -10 & $(8,0) 2,0 ; 2$ & 83.45 \\
\hline & & $(4,2) 2,0 ; 2$ & 5.68 \\
\hline & & $(0,4) 2,0 ; 2$ & 6.71 \\
\hline & & $(2,0) 2,0 ; 2$ & 4.16 \\
\hline \multirow{3}{*}{$\begin{array}{c}4_{1}^{+} \\
|2[1][0] 4,0 ; 4\rangle\end{array}$} & -10 & $(8,0) 4,0 ; 4$ & 40.86 \\
\hline & & $(4,2) 4,0 ; 4$ & 53.81 \\
\hline & & $(0,4) 4,0 ; 4$ & 5.33 \\
\hline \multirow{3}{*}{$\begin{array}{c}0_{2}^{+} \\
|2[1][0] 0,0 ; 0\rangle\end{array}$} & -10 & $(4,2) 0,0 ; 0$ & 77.78 \\
\hline & & $(0,4) 0,0 ; 0$ & 11.11 \\
\hline & & $(2,0) 0,0 ; 0$ & 11.11 \\
\hline \multirow{4}{*}{$\begin{array}{c}0_{3}^{+} \\
|2[1][0] 0,0 ; 0\rangle\end{array}$} & -10 & $(8,0) 0,0 ; 0$ & 1.13 \\
\hline & & $(4,2) 0,0 ; 0$ & 77.92 \\
\hline & & $(0,4) 0,0 ; 0$ & 10.54 \\
\hline & & $(2,0) 0,0 ; 0$ & 10.41 \\
\hline \multirow{4}{*}{$\begin{array}{c}0_{4}^{+} \\
|2[1][0] 0,0 ; 0\rangle\end{array}$} & -10 & $(8,0) 0,0 ; 0$ & 16.87 \\
\hline & & $(4,2) 0,0 ; 0$ & 79.86 \\
\hline & & $(0,4) 0,0 ; 0$ & 2.57 \\
\hline & & $(2,0) 0,0 ; 0$ & 0.70 \\
\hline \multirow{4}{*}{$\begin{array}{c}2_{2}^{+} \\
|2[1][0] 2,0 ; 2\rangle\end{array}$} & -10 & $(8,0) 2,0 ; 2$ & 1.41 \\
\hline & & $(4,2) 2,0 ; 2$ & 72.57 \\
\hline & & $(0,4) 2,0 ; 2$ & 15.74 \\
\hline & & $(2,0) 2,0 ; 2$ & 10.28 \\
\hline \multirow{3}{*}{$\begin{array}{c}2_{3}^{+} \\
|2[1][0] 0,2 ; 2\rangle\end{array}$} & -10 & $(4,2) 0,2 ; 2$ & 77.78 \\
\hline & & $(0,4) 0,2 ; 2$ & 11.11 \\
\hline & & $(2,0) 0,2 ; 2$ & 11.11 \\
\hline
\end{tabular}

(a)

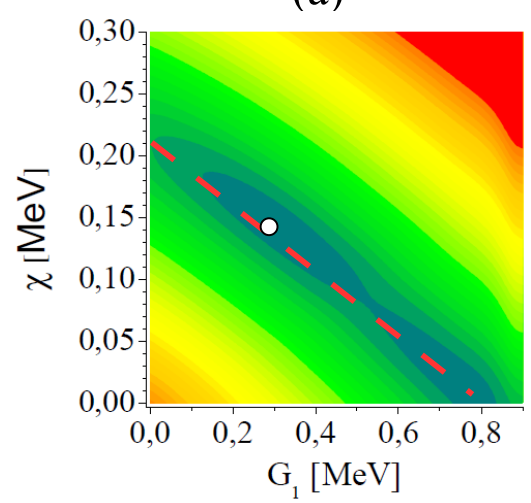

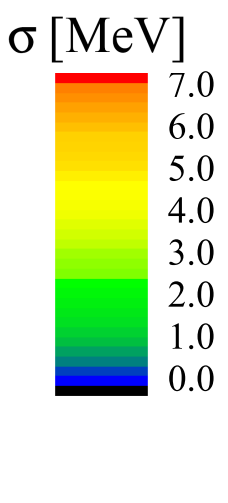

(b)

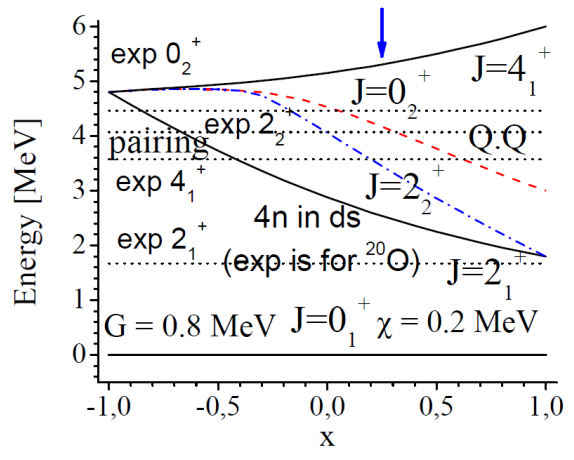

Figure 2. (Color online) Result for ${ }^{20} \mathrm{O}$ with the Hamiltonian (7). (a) The absolute RMS deviation $\sigma$ in $\mathrm{MeV}$ for the excitation spectrum in the $d s$-shell, calculated in full SU(3) basis. The white circle denotes the position where $\sigma$ is minimal. (b) Excitation spectrum of the lowest-lying energies with the control parameter $x$ varying from -1 to 1 along the red line in part (a).

serve as a measure of the influence of each of the terms from the residual interactions on the energy spectra of the considered nucleus. An interesting conclusion from these two-parameter figures is that similar results for $\sigma$ can be obtained by using various pairs of values $(\chi, G)$. This cor- responds to somewhat different spectra in all these cases — from purely rotational to somewhat more pairing-like modes.

At the pairing limit $(x=-1)$, a non-degenerate $v=0$, $J=0$ state is separated by the degenerated rest of $v=2$, 
(a)

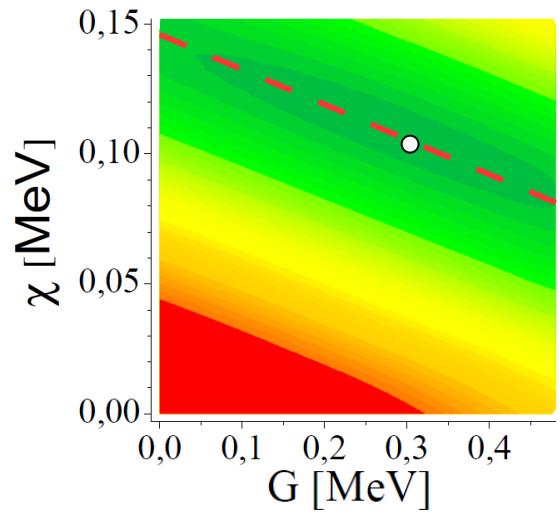

(c)

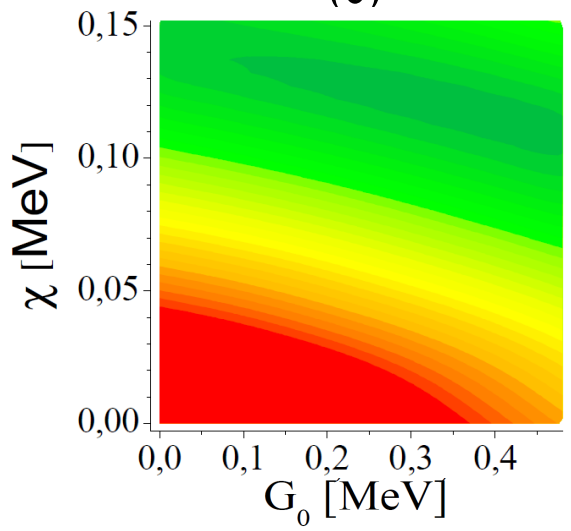

(b)

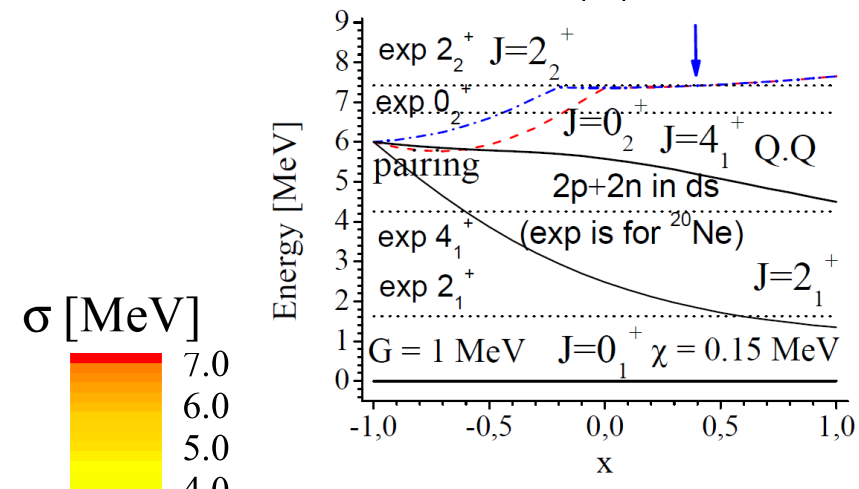

(d)

2.0

1.0

0.0

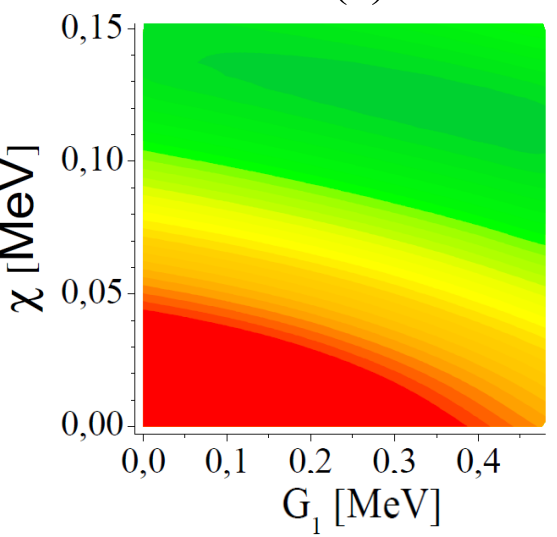

Figure 3. (Color online) Similar to Fig. 2 but for the nucleus ${ }^{20} \mathrm{Ne}$. Calculations are performed when (a) the total pairing or just (c) the isoscalar or (d) the isovector part of the pairing is included in the Hamiltonian. Part (b): the excitation energies obtained with the Hamiltonian (5) with the control parameter $x$ varying from -1 to 1 along the red line in part (a).

$J=0_{1}^{+}, 2_{1}^{+}, 2_{2}^{+}, 4_{1}^{+}$states by the pairing gap $2 \Delta=G \Omega$. Very soon after the pairing limit, for non-vanishing values of $\chi$ first the lowest $J=2_{1}^{+}$is separated from the rest of the degenerated excited states, then around $x=0$ all the states degeneracy is removed and the triplet of $v=2, J=2_{1}^{+}, 0_{2}^{+}, 4_{1}^{+}$ is clearly observed, which reproduces the spectra typical for the quadrupole phonon model [11] as well as in the Interacting Boson Model [12]. In the pure SU(3) limit ( $x=1)$, the rotational sequence of $v=2, J=0_{1}^{+}, 2_{1}^{+}, 4_{1}^{+}$states in the ground band is recovered, based on the leading SU(3) irrep $(8,0)$ (see Table 1). As a result, a degeneracy of the two $v=2, J=2^{+}$appears, which is lifted for nonvanishing values of $G$. The second $v=2, J=0_{2}^{+}$, based on leading $\operatorname{SU}(3)$ irrep $(4,2)$, is the band head of the excited $0_{2}^{+}$. It is obvious that the complicated spectra observed in real nuclear systems are best reproduced by taking into account both the pairing and quadrupole-quadrupole interactions.

In the case of ${ }^{20} \mathrm{Ne}$ (see Fig. 3), the RMS estimate is performed over the 21 lowest-lying positive-parity experimental energies $E_{E x p}^{i}$. The results are given for the three choices of the pairing interaction - the isoscalar, the isovector and the total pairing with a common strength parameter value. For this nucleus we obtain a more rotational spectrum but again observe a flat area of minima with similar RMS values of $\sigma$. Compared to the ${ }^{20} \mathrm{O}$ case, here the region of values suggesting good description of the experiment do not reach the pure-pairing side. Also the slope of change is smaller and the point of the best description shifts to the right towards a more rotational spectrum (see the position of the blue arrow in Fig. 2(b) compared to the Fig. 3(b)).

\section{Three-parameter estimate and the phase transitions}

Further, we can separate the two pairing modes - the isoscalar and the isovector one - and use a Hamiltonian of the form

$$
H=G_{0} V_{P_{\mathrm{isc}}}+G_{1} V_{P_{\mathrm{iv}}}-\frac{\chi}{2} Q \cdot Q .
$$

In this case, one has to introduce two control parameters $y$ and $z$, described in Sec. 3.2 of Ref. [2] (see also [13]). These are defined as having the following relation with the three strengths $G_{0}, G_{1}$ and $\chi: y=\chi /\left(\chi+G_{1}\right)$, $z=\left(\chi+G_{1}\right) /\left(\chi+G_{0}+G_{1}\right)$ and the scaling parameter $c=\chi+G_{0}+G_{1}$. Using them, the Hamiltonian becomes

$$
H=c(1-z) V_{P_{\mathrm{isc}}}+c(1-y) z V_{P_{\mathrm{iv}}}-c y z Q \cdot Q .
$$




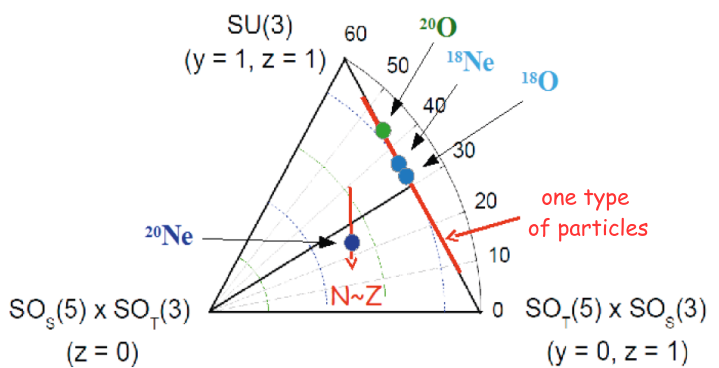

Figure 4. (Color online) A symmetry triangle that illustrates the dominance of one of the interactions: quadrupole-quadrupole, isoscalar pairing or isovector pairing. The coordinates of a point of interest are $y$ and $z$. The four circles locate the results for the nuclei ${ }^{18} \mathrm{Ne},{ }^{18} \mathrm{O},{ }^{20} \mathrm{Ne}$, and ${ }^{20} \mathrm{O}$.

The ratio between the best-fit values for the parameters $\chi, G_{0}$ and $G_{1}$ can be plot on a diagram resembling the Casten symmetry triangle, where each vertex represents one of the modes in pure form. The two control parameters $y$ and $z$ have the following meaning: an angle and a distance from the point of interest to one of the vertices, respectively.

The corresponding three-parameter outcome is shown in Figs. 4 and 5. The best three-parameter results in the case of ${ }^{20} \mathrm{Ne}$ are obtained for the values $\chi=0.11 \mathrm{MeV}$, $G_{0}=0.28 \mathrm{MeV}$, and $G_{1}=0.28 \mathrm{MeV}$. The other three nuclei in the study $\left({ }^{18} \mathrm{O},{ }^{18} \mathrm{Ne}\right.$, and $\left.{ }^{20} \mathrm{O}\right)$ have only one type of valence particles (protons or neutrons), so the threeparameter investigation is not applicable to them. We see that the best results are obtained for $G_{0}$ values close to the ones of $G_{1}$.

In Fig. 4, the results obtained for all 4 nuclei in our study are presented. The outcome for the nuclei ${ }^{18} \mathrm{O}$ and ${ }^{18} \mathrm{Ne}$ has been obtained as the best two-parameter estimate using the Hamiltonian (7). One can see that only the ${ }^{20} \mathrm{Ne}$ result for the parameters lies inside the triangle. The cases of no isoscalar pairing allowed (which is when only one type of valence particles is present in the system) lie along the $\mathrm{SO}_{\mathrm{T}}(5)-\mathrm{SU}(3)$ line. Moreover, the more collective ${ }^{20} \mathrm{O}$ nucleus is positioned closer to the $\mathrm{SU}(3)$ vertex.

Finally, in Fig. 5, a comparison between excitation spectra calculated for ${ }^{20} \mathrm{Ne}$ has been done. It can be seen that the deviation from the experimental energy spectrum is reduced once one goes from two-parameter to threeparameter description. Also, the degeneracy of the states $0_{2}^{+}, 0_{3}^{+}, 2_{2}^{+}$and $2_{3}^{+}$is removed as is in the experiment.

\section{Conclusion}

The relation between the pairing and the quadrupole interaction established [1,2] on the basis of their complementarity to the Wigner's spin-isospin $\mathrm{U}_{\mathrm{ST}}(4)$ symmetry was used to elucidate the algebraic structure of an extended Pairing-plus-Quadrupole Model, realized in the framework of the Elliott's SU(3) scheme [3]. The pairing part of the Hamiltonian consists of $p p$-, $n n$ - and $p n$-pairing terms. The relationship between the basis states classified in each of the dynamical symmetries is obtained, which allows for the evaluation of the matrix elements of the operators representing each algebra in the reductions. This approach is used to study the combined effects of the quadrupolequadrupole and pairing interactions on the energy spectra of the nuclear systems. In a forthcoming article [14], we will describe all these effects in a sequence of $N=Z$ nuclei with $A=2$ and 4 nucleons. There, results for $B(M 1)$ and $B(E 2)$ transition strengths will also be displayed and probably included in the best fit estimate.

The phases as dynamical symmetries of an extended Pairing-plus-Quadrupole Model are investigated in the framework of the Elliott's SU(3) scheme. The phase transitions between all three limits are studied by evaluating the weights of the different interactions in the PQM Hamiltonian for several nuclear systems with 2 and 4 particles in the $d s$-shell. The probability distributions $\left|C_{i j}\right|^{2}$ (transformation brackets) with which the states of the SU(3) basis enter into the expansion of the pairing basis are obtained numerically. In this way, the importance (weight) of the different SU(3) - states can be used, when we need to impose restrictions on the basis because of computational difficulties. The parameter adjustment for realistic nuclear systems gives the influence of each of the considered pairing and quadrupole modes on the reproduction of the nuclear spectra. This evaluation is achieved and clarified by the introduction of two or three control parameters. The comparison of the results for different numbers and types of particles that constitute the valence shell for the nuclear system could be compared to evaluate the onset of deformation.

\section{Acknowledgements}

K.P.D. is grateful for the hospitality of the BLTP of JINR, Dubna. This work was also partially supported by the Bulgarian National Foundation for Scientific Research under Grant No. DFNI-026/12.12.2014.

\section{References}

[1] K.P. Drumev and A.I. Georgieva, Proc. of 32-nd Int. Workshop on Nuclear Theory (IWNT-32), eds. A.I. Georgieva, N. Minkov (Heron Press, Sofia, 2013), 151.

[2] A.I. Georgieva and K.P. Drumev, Eur. Phys. J. Web Conf., this issue.

[3] J.P. Elliott and M. Harvey, Proc. Roy. Soc. London, Ser. A 272, 557 (1963); J.P. Elliott and C.E. Wilsdon, Proc. Roy. Soc. London, Ser. A 302, 509 (1968)

[4] C.E. Vargas, J.G. Hirsch, J.P. Draayer, Nucl. Phys. A 690, 409 (2001)

[5] J.P. Draayer, Y. Leschber, S.C. Park, R. Lopez, Comp. Phys. Commun. 56, 279 (1989) 


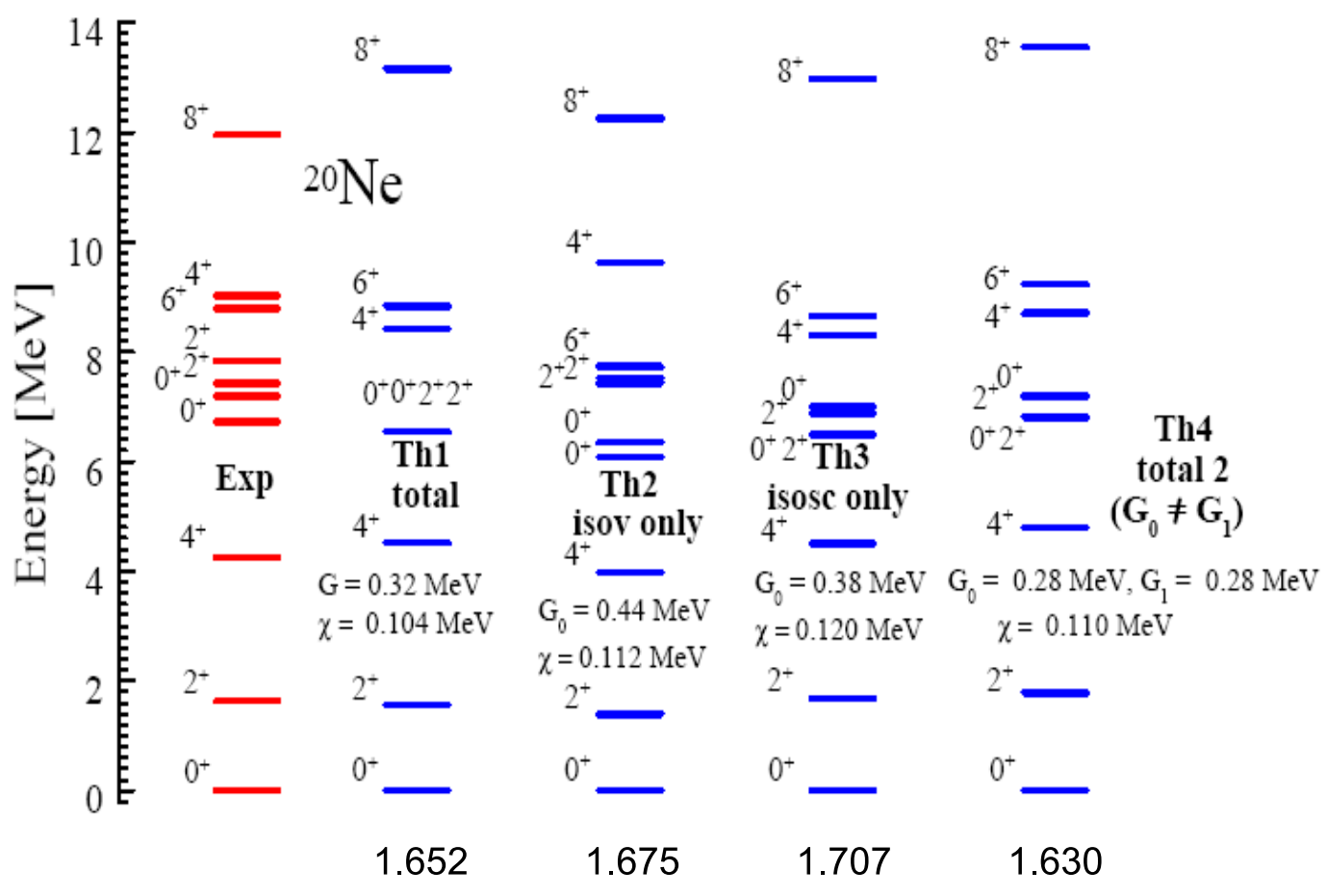

Figure 5. (Color online) Comparison of the experimental and theoretical spectra obtained for ${ }^{20} \mathrm{Ne}$ with the Hamiltonians (5), (6), (7), and (8). Below each spectrum, the RMS value obtained is written.

[6] C. Bahri and J.P. Draayer, Comp. Phys. Commun. 83, 59 (1994)

[7] C. Bahri, J. Escher, J.P. Draayer, Nucl. Phys. A 592, $171(1995)$

[8] O. Castaños, J.P. Draayer, Y. Leschber, Z. Phys. A 239, 33 (1988)

[9] V.K.B. Kota and J.A. Castilho Alcarás, Nucl. Phys. A 764, 181 (2006)
[10] National Nuclear Data Center, [http://www.nndc.bnl.gov].

[11] D. Janssen, R.V. Jolos, F. Dönau, Nucl. Phys. A 224, 93 (1974)

[12] A. Arima and F. Iachello, Ann. Phys. 99, 253 (1976)

[13] J. Cseh and J. Darai, arXiv:1404.3503v1[nucl-th] (2014)

[14] K.P. Drumev and A.I. Georgieva, in preparation. 